\title{
Contribution of the intra- and intermolecular routes in autocatalytic zymogen activation: application to pepsinogen activation
}

\author{
Ramón Varón ${ }^{1 凶}$, Matilde E. Fuentes ${ }^{1}$, Manuela García-Morenoํㅜ Francisco \\ Garcia-Sevilla ${ }^{1}$, Enrique Arias ${ }^{2}$, Edelmira Valero ${ }^{1}$ and Enrique Arribas ${ }^{3}$ \\ ${ }^{1}$ Grupo de Modelización en Bioquímica, Departamento de Química-Física, and ${ }^{2}$ Departamento de Informática, \\ and ${ }^{3}$ Departamento de Física Aplicada, Escuela Politécnica Superior de Albacete, Universidad de Castilla-La

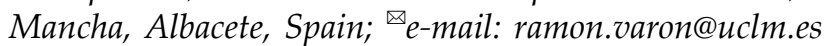

Received: 30 November, 2005; revised: 10 April, 2006; accepted: 05 May, 2006 available on-line: 12 June, 2006

\begin{abstract}
Taking as the starting point a recently suggested reaction scheme for zymogen activation involving intra- and intermolecular routes and the enzyme-zymogen complex, we carry out a complete analysis of the relative contribution of both routes in the process. This analysis suggests the definition of new dimensionless parameters allowing the elaboration, from the values of the rate constants and initial conditions, of the time course of the contribution of the two routes. The procedure mentioned above related to a concrete reaction scheme is extrapolated to any other model of autocatalytic zymogen activation involving intra- and intermolecular routes. Finally, we discuss the contribution of both of the activating routes in pepsinogen activation into pepsin using the values of the kinetic parameters given in the literature.
\end{abstract}

Keywords: enzyme kinetics, autocatalysis, proenzyme activation, intermolecular activation, intramolecular activation

\section{INTRODUCTION}

Proteolytic enzymes are normally synthesized and secreted as inactive precursors, which are activated at a physiologically appropriate time and place, in order to protect cells which produce them. These precursors are known as proenzymes or zymogens. They must undergo an activation process, usually a limited proteolysis, to attain their catalytic activity. Understanding of this very important phenomenon is crucial to full elucidation of the behaviour of many fundamental physiological processes, such as food digestion (Chen et al., 2003), cell death (Boatright \& Salvesen, 2003; Shi, 2004), differentiation (Pearton et al., 2001), immune response (Kanost et al., 2004), blood coagulation (Spronk et al., 2003), fibrinolysis (Varón et al., 1986; Marin et al., 2003) and response to injury (Lluis et al., 2001). Other examples are the evolution of viruses, oncogenes and metastatic cells (McKay et al., 2003).

Proenzyme activation by proteolytic cleavage of one or more peptide bonds requires the presence of an activating enzyme. In those cases in which the activating enzyme is the same as the activated one, the proenzyme activation process is called autocatalytic. Physiological examples are the activation of trypsinogen into trypsin (Manjabacas et al., 1995; Liu \& Wang, 2004), the conversion of pepsinogen into pepsin (EC 3.4.23.1) (Al-Janabi et al., 1972; Richter et al., 1998; Fuentes et al., 2005a; 2005b), and prekallikrein into kallikrein (Magklara et al., 2003; ShariatMadar et al., 2004).

Several experimental and theoretical models of autocatalysis have been extensively investigated. These include the kinetic behaviour of the autocatalytic activation of a zymogen in the absence of any ligand (inhibitor or substrate) of the activating/active enzyme (Varón et al., 1990; García-Moreno et al., 1991; Wu et al., 2001), in the presence of a substrate of the enzyme yielding a chromophoric product (Varón et al., 1991; 1992) and also in the presence of an inhibitor of the enzyme (Manjabacas et al., 1992; 1995; 1996; 2002). However, in these studies the zymogen was considered to have no enzyme activity. 
Nevertheless, references to the intramolecular activation of zymogens are increasingly more frequent (Lin et al., 1992; Tanaka \& Yada, 1997; Richter et al., 1999).

Al-Janabi et al. (1972) offered kinetic evidence for the existence of two activation pathways (intraand intermolecular) for the activation of pepsinogen to pepsin, suggesting a highly simplified reaction mechanism shown in Scheme 1 , where $Z$ is pepsinogen, $E$ is pepsin and $W$ the peptide released from $Z$.

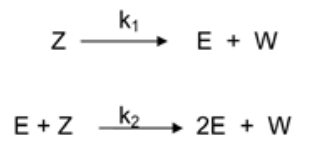

Scheme 1

(b)

Recently, Fuentes et al. (2005a) suggested a general mechanism, shown in Scheme 2, applicable to any zymogen activation, for which they carried out a kinetic analysis and suggested an experimental design and a kinetic data analysis to evaluate the rate constants $k_{1}$ and $k_{3}$ and the Michaelis constant. Then they applied these constant values to the kinetic characterization of pepsinogen activation.

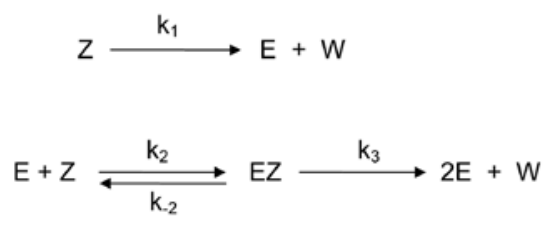

Scheme 2

The above mechanism exhibits simultaneously two catalytic routes, an intramolecular activation process, route $(a)$, and an autocatalytic zymogen activation process catalyzed by the same enzyme it produces, route $(b)$. This mechanism includes the reversible formation of an intermediary active enzyme-zymogen complex in the intermolecular activation step.

In a more recent contribution (Fuentes et al., 2005b) a chromogenic substrate of the active enzyme is included in reaction medium. The mechanism proposed is shown in Scheme 3. It consists in the addition of the coupled route (c) corresponding to the catalytic action of the activated enzyme, $E$, on a chromogenic substrate, so that the time course of the substrate and/or the product of this step can be experimentally followed using a continuous procedure. Using this measurement procedure and assuming rapid equilibrium of the two reversible steps they obtained the values of the rate constants $k_{1}$ and $k_{3}$ and the equilibrium constant $K_{2}$ involved in Scheme 3 at $\mathrm{pH}=3.0$ and $25^{\circ} \mathrm{C}$ temperature.

In all of the contributions mentioned above the main objective of the kinetic analysis was the determination of the kinetic parameters involved in the

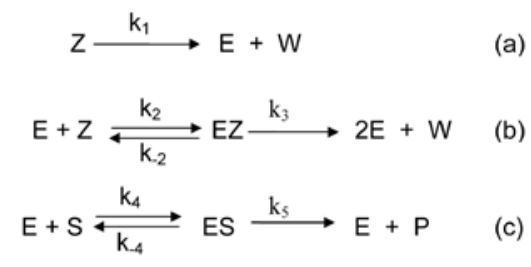

Scheme 3

reaction mechanisms proposed in each case, and no theoretical analysis of the participation in the process of the intra- and intermolecular zymogen activation routes valid for the whole reaction course have been carried out and only some conclusions from the experimental results were obtained.

In some contributions, the intra- and intermolecular routes in autocatalytic zymogen activation have only been described from experimental results (Al-Janabi et al., 1972; McPhie, 1974; Marciniszyn et al., 1976; Richter et al., 1999). Only in a recent contribution of Fuentes et al. (2005b), an individualised theoretical analysis of the partition ratio between the two routes in Scheme 3 has been carried out. Nevertheless, this analysis has some limitations that we will discuss below.

Therefore, the aims of the present paper are: 1) To perform a complete kinetic analysis and to suggest a new dimensionless parameter giving the contribution, to the activation process, at any reaction time, of both activation routes, i.e. the intra- and intermolecular ones in an zymogen activation process evolving according to the reaction mechanism in Scheme 2; (2) To extend the above procedure to any zymogen activation process such as those described by Schemes 1 and 3; (3) To study the effect on these parameters of the initial concentrations; (4) To apply the results obtained to the pepsinogen autoactivation into pepsin using the kinetic parameter values obtained in the literature; (5) To suggest a kinetic data analysis and an experimental design to evaluate $k_{2}$ and $k_{-2}$ corresponding to the pepsinogen activation according to Scheme 2; (6) To review the previous analysis for the partition ratio between the uni- and bimolecular activation routes in Scheme 3.

\section{MATERIALS AND METHODS}

Simulated progress curves were obtained by numerical integration of a set of differential equations describing the kinetic behaviour of the evolving reaction according to the corresponding mechanism under study, using values of the rate constants and initial concentrations either published in the literature or arbitrary, but reasonable. This numerical solution was found by using the classical fourth-order Runge-Kutta formula, but applying an adaptative stepsize control originally invented by Fehlberg 
(Fehlberg, 1970; Mathews \& Fink, 1999) using the computer program WES, implemented in Visual C++ 6.0 for MS Windows and which allows one to simulate the kinetic behaviour of enzyme reactions with up to 32 species (García-Sevilla et al., 2000). The above program was run on a PC compatible computer based on a Pentium IV/2 GHz processor with 512 Mbytes of RAM.

The plots of the data obtained from the numerical integration as well as the plot of equation (50) and the fittings to exponential equation (55) done in Fig. 5 were carried out using the SigmaPlot Scientific Graphing System, version 8.02 (2002, SPSS Inc).

$\left[E_{T}\right]_{u}$ and $\left[E_{T}\right]_{a}$ involved in equation (7) are obtained as explained in the Theory section.

The experimental data necessary for the application of these analyses were taken from previous contributions from our group (Fuentes et al., 2005a; 2005b) and from other authors (Al-Janabi et al., 1972).

\section{KINETIC ANALYSIS OF THE RELATIVE PREVALENCE OF THE ACTIVATION ROUTES}

This section is entirely devoted to an analysis of the relative contribution of both activation routes in Scheme 2. Then, as part of the Results and Discussion section we will extend the analysis to other more or less complex zymogen activation mechanisms. We will suggest new dimensionless parameters needed to establish the relative contribution of both activation routes $(a)$ and $(b)$ in Scheme 2 . Some of these parameters are concentration-based and others are rate-based.

\section{Notation and definitions}

In the following we summarize some notation and definitions, some of them introduced here for the first time and all of them necessary to facilitate the analysis below.

$[E],[Z],[E Z]$ : Instantaneous concentrations of the species $E, Z$ and $E Z$, respectively.

$[E]_{0^{\prime}},[Z]_{0^{\prime}}[E Z]_{0}$ : Initial concentrations of the species $E, Z$ and $E Z$, respectively.

$K_{m}$ : The Michaelis-Menten constant for the activation of zymogen towards its active enzyme, i.e.:

$$
K_{m}=\frac{k_{-2}+k_{3}}{k_{2}}
$$
plex, i.e.:
$K_{2}=\frac{k_{-2}}{k_{2}}$

$\left[E_{T}\right]_{a}$ : Total active enzyme concentration at time $t$ derived from zymogen activation, i.e.:

$\left[E_{T}\right]_{a}=[E]+[E]-[E]_{0}$

$\eta$ : Dimensionless parameter defined as the fraction of $[Z]_{0}$ activated to the active enzyme $E$, i.e.:

$\eta=\frac{[E]-[E]_{0}}{[Z]_{0}}$

Note that when all the zymogen, $Z$, has been activated to the enzyme, $E$, then $\eta=1$.

$t_{\infty}$ : The time from which the zymogen activation is complete. Strictly, this time is equal to the time in which $\eta=1$. Nevertheless, since reaching the value $\eta=1$ can be too high or difficult to know, due to experimental difficulties, the reaction can be considered complete for an $\eta$-value less but near unity, e.g. $\eta=0.9999$. Thus, $t_{\infty}$ is the time in which the reaction reaches $\eta=1$ or any arbitrarily chosen value near unity that we will denote as $\eta_{\infty}$. In this work we always considered $\eta_{\infty}=0.9999$.

$\left[E_{T}\right]_{u}$ : Total active enzyme concentration at time $t$ derived from the unimolecular (intramolecular) route, i.e.:

$$
\left[E_{T}\right]_{u}=\int_{0}^{t^{\prime}} k_{1}[Z] d t
$$

where $t^{\prime}$ is any reaction time which in the resulting integral must be replaced by $t$.

$\left[E_{T}\right]_{b}$ : Total active enzyme concentration at time $t$ derived from the bimolecular (intermolecular) route, i.e.:

$\left[E_{T}\right]_{b}=\left[E_{T}\right]_{a}-\left[E_{T}\right]_{u}$

Suggested dimensionless parameters giving the relative prevalence of both activation routes.

The fraction of $\left[E_{T}\right]_{a}$ derived from the intramolecular route, which we denote as $R_{u^{\prime}}$ is:

$R_{u}=\frac{\left[E_{T}\right]_{u}}{\left[E_{T}\right]_{a}}$

Likewise, the fraction of $\left[E_{T}\right]_{a}$ from the intermolecular route, which we denote as $R_{b}$, is

$R_{b}=\frac{\left[E_{T}\right]_{b}}{\left[E_{T}\right]_{a}}$ 

have:

Obviously, from equations (6), (7) and (8) we $R_{u}+R_{b}=1$

Next, we define a dimensionless kinetic parameter, $R$, giving the relative contributions to the activation process of both routes $(a)$ and $(b)$ as the partition ratio:

$$
R=\frac{R_{u}}{R_{b}}
$$

From equations (9) and (10), $R$ can be written as a function of $R_{u}$ only or as a function of $R_{b}$ only by means of:

$$
R=\frac{R_{u}}{1-R_{u}}
$$

$$
\begin{aligned}
& \text { or } \\
& R=\frac{1-R_{b}}{R_{b}}
\end{aligned}
$$

Any of the above defined dimensionless parameters $R_{u^{\prime}} R_{b}$ and $R$ can be used to know the relative weight of the intra- and intermolecular-activation routes and from any of these parameters the other two are immediately determined from equations (10)-(12).

The above-defined parameter $R_{u}$ furnishes the relative weight of the unimolecular and bimolecular activation routes from $t=0$ to any reaction time $t$ based on the concentrations. Depending whether $R_{u}$ $>0.5, R_{u}=0.5$ or $R_{u}<0.5$, at the reaction time considered, the unimolecular route will prevail over the bimolecular one, both routes will be balanced, or the bimolecular route will prevail over the unimolecular one, respectively.

This participation of the intramolecular route can be also expressed as the percentage of the total active enzyme derived from the zymogen activations as $100 R_{u}$

In the following, we will denote as $R_{u, 0}$ the $R_{u}$ value at the onset of the reaction and as $R_{u, \infty}$ the $R_{u}$ value at the end of the reaction, i.e.:

$$
\begin{aligned}
& R_{u, 0}=\lim _{t \rightarrow 0} R_{u} \\
& R_{u, \infty}=\lim _{t \rightarrow \infty} R_{u}
\end{aligned}
$$

Evaluation of $R_{u}$

In order to obtain analytical equation [e.g. equation (45) below] from equation (7), giving the time course of $R_{u}$ during the whole course of the re- action, the corresponding analytical expressions for [Z] [e.g. equation (40) below] and $\left[E_{T}\right]_{a}$ [e.g. equation (43) below] valid during the whole course of the reaction are required. But this is not the case concerning Scheme 2.

The values of $R_{u}$ at any reaction time (including obviously those of $R_{u, 0}$ and $R_{u, \infty}$ ) can be obtained from numerical integration as follows. The kinetic behaviour of species $Z, E$ and $E Z$ involved in the mechanism shown in Scheme 2 is described by the following set of differential equations:

$$
\begin{aligned}
& \frac{d[Z]}{d t}=-k_{1}[Z]-k_{2}[Z][E]+k_{-2}[E Z] \\
& \frac{d[E]}{d t}=k_{1}[Z]-k_{2}[Z][E]+\left(k_{-2}+2 k_{3}\right)[E Z] \\
& \frac{d[E Z]}{d t}=k_{2}[Z][E]-\left(k_{-2}+k_{3}\right)[E Z]
\end{aligned}
$$

To evaluate the integral in equation (5) we can merely add, to the above set of differential equations, the equation:

$$
\frac{d\left[E_{T}\right]_{u}}{d t}=k_{1}[Z]
$$

The differential equations (15)-(18) constitute a non-linear system that does not allow any analytical solution. However, the solution can be obtained by numerical integration by applying any integration method. Numerical integration of the above set of differential equation furnishes the time course of $[Z],[E],[E Z]$ and $\left[E_{T}\right]_{u}$. From the values of $[E]$ and $[E Z]-v a l u e s$ at any time the $\left[E_{T}\right]_{a}-$ value (i.e., $[E]+$ $\left.[E Z]-[E]_{0}\right)$ is immediately obtained. The program WES has an option yielding also the sum $[E]+[E Z]$. In Fig. 1, as an example, we show the result of a numerical integration of the set of differential equations (15)-(18) for the same values of the rate constants and initial zymogen concentration used by Fuentes et al. (2005). According to equation (7), dividing the instantaneous values of $\left[E_{T}\right]_{u}$ and $\left[E_{T}\right]_{a^{\prime}}$ the time course of $R_{u}$ is obtained. In those cases where $R_{u, \infty}$ $<0.5$ there exists a time, which we will denote as $\theta$, at which $R_{u}=0.5$ and therefore from time $t=0$ until $t=\theta$ the intramolecular route prevails and after this time the intermolecular route prevails. In Fig. 2 we show the time course of $R_{u}$ for the same values of the rate constants as in Fig. 1 and different values of the initial zymogen and enzyme concentrations.

In spite of the fact that no analytical expression of $R_{u}$ valid for the whole course of the reaction exists, an analytical expression can be obtained for 

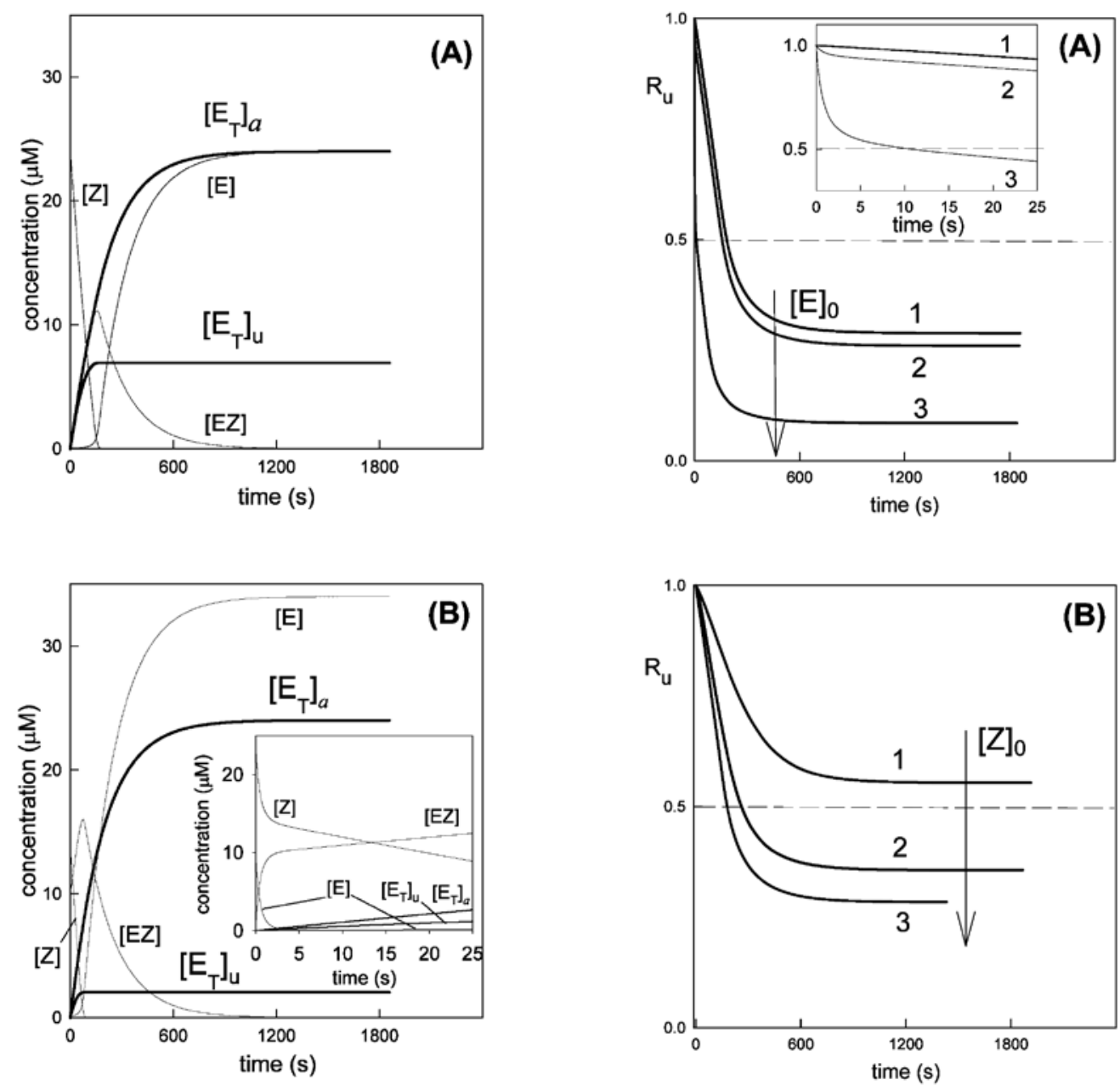

Figure 1. Simulated progress curves of $[Z],[E],[E Z],\left[E_{T}\right]_{u}$ and $[E T]_{a}$ obtained from numerical integration of the set of differential equations (15)-(18).

$\left[E_{T}\right]_{a}$ is obtained from equation (3) either apart or using the corresponding option of the software WES. The reaction is considered finished when $\eta_{\infty}=0.9999$. The following values of the rate constants and initial zymogen concentration were arbitrarily chosen for the integration: $k_{1}=$ $4.0 \times 10^{-3} \mathrm{~s}^{-1}, k_{2}=1.0 \times 10^{3} \mathrm{M}^{-1} \mathrm{~s}^{-1}, k_{-2}=2.1 \times 10^{-4} \mathrm{~s}^{-1}, k_{3}=$ $5.4 \times 10^{-4} \mathrm{~s}^{-1}$ and $[Z]_{0}=24 \mu \mathrm{M}$ and the values of the initial enzyme concentrations were in (A) $[E]_{0}=0$ and in (B) $[E]_{0}$ $=10 \mu \mathrm{M}$. The values of the time for which $\eta=\eta_{\infty}$ were $1859 \mathrm{~s}$ in (A) and $1857 \mathrm{~s}$ in (B). The inset shows details in the first $25 \mathrm{~s}$ of the reaction.

$R_{u}$ valid only for the first instant of the reaction. Effectively, at a short reaction time, $t(t \rightarrow 0)$, from the onset of the reaction, the instantaneous concentrations of both the zymogen, $Z$, and the free enzyme, $E$, remain near their initial concentrations, i.e.

$[Z] \approx[Z]_{0} \quad(t \rightarrow 0)$

and

$[E] \approx[E]_{0} \quad(t \rightarrow 0)$

During this short reaction time, according to equation (18), it is observed that:

$[E T]_{\mathrm{u}} \approx k_{1}[Z]_{0} t \quad(t \rightarrow 0)$

Figure 2. Time course of $R_{u}$ corresponding to Scheme 2 obtained from simulated progress curves of $\left[E_{T}\right]_{u}$ and $\left[E_{T}\right]_{a}$ for the same values of the rate constants as in Fig. 1 .

The reaction is considered finished when $\eta_{\infty}=0.9999$. (A) In all cases $[Z]_{0}=24 \mu \mathrm{M}$ and in curves 1,2 and 3 the $[E]_{0}$ values were 0,1 and $10 \mu \mathrm{M}$. The values of $R_{u, \infty} \theta$ and $t_{\infty}$ in curves 1,2 , and 3 are given in Table 1 . (B) In all cases $[E]_{0}=0.01 \mu \mathrm{M}$ and in curves 1,2 and 3 the $[Z]_{0}$ values were $0.1,1$ and $10 \mu \mathrm{M}$. The values of $R_{u, \infty} \theta$ and $t_{\infty}$ in curves 1, 2 and 3 are given in Table 1 . The inset shows details in the first $25 \mathrm{~s}$ of the reaction.

Hence, adding side by side differential equations (16) and (17) and taking into account equation (3) the following equivalence is obtained:

$\frac{d\left[E_{T}\right]_{a}}{d t} \approx k_{1}[Z]_{0}+k_{3}[E Z] \quad(t \rightarrow 0)$

An approximate expression of [EZ] valid for very short reaction time can be derived from equation (17) inserting in it equations (19) and (20) and integrating the following resulting linear differential equation:

$\frac{d[E Z]}{d t} \approx k_{2}[Z]_{0}[E]_{0}-\left(k_{-2}+k_{3}\right)[E Z](t \rightarrow 0)$ 
with the initial condition $[E Z]_{0}=0$. The result is

$[E Z] \approx \frac{k_{2}[E]_{0}[Z]_{0}}{k_{-2}+k_{3}}\left\{1-e^{-\left(k_{-2}+k_{3}\right) t}\right\} \quad(t \rightarrow 0)$

If this expression of [EZ] is inserted into equation (22), we have, after integration:

$$
\begin{aligned}
& {\left[E_{T}\right]_{a} \approx\left(k_{1}+\frac{k_{2} k_{3}[E]_{0}}{k_{-2}+k_{3}}\right)[Z]_{0} t+} \\
& +\frac{k_{2} k_{3}[Z]_{0}[E]_{0}}{\left(k_{-2}+k_{3}\right)^{2}}\left\{e^{-\left(k_{-2}+k_{3}\right) t}-1\right\} \quad(t \rightarrow 0)
\end{aligned}
$$

If equations (21) and (25) are inserted now into equation (7) it results in the following approached analytical equation for the time course of $R_{u}$ from $t=0$ to a very short reaction time, $t$-value:

$$
\begin{aligned}
R_{u} \approx & k_{1}[Z]_{0} t /\left\{\left(k_{1}+\frac{k_{2} k_{3}[E]_{0}}{k_{-2}+k_{3}}\right)[Z]_{0} t+\right. \\
& \left.+\frac{k_{2} k_{3}[Z]_{0}[E]_{0}}{\left(k_{-2}+k_{3}\right)^{2}}\left(e^{-\left(k_{-2}+k_{3}\right) t}-1\right)\right\} \quad(t \rightarrow 0)
\end{aligned}
$$

The approached equation (26) is only rigorously valid at $t=0$ and in this case both the numerical value of $R_{u}$ (i.e. $R_{u, 0}$ ) and that setting $t=0$ in equation (26) coincide, and they are equal to unity. Thus, we have shown that in the mechanism in Scheme 2 , independently of the $[Z]_{0}$ and $[E]_{0}$ values, it is observed that:

$R_{u, 0}=1$

We have been not able to find equivalent reasoning to derive an approached analytical equation for $R_{u, \infty}$ [Note that equation (26) is only valid for low $\mathrm{t}$-values]. We only can state that:

$0<R_{u, \infty}<1$

\section{RESULTS AND DISCUSSION}

Previously, kinetic analyses of the reactions whereby a zymogen is simultaneously activated through both a uni (intra)- and a bi (inter)-molecular routes by the action of the active enzyme were performed and used for the experimental determination of the kinetic parameters involved in the activation reaction (Al-Janabi et al., 1972; Glick et al., 1989; Lin et al., 1992; Richter et al., 1999; Fuentes et al., 2005a; 2005b). However, as far as we know, none of the above contributions carried out any complete kinetic analysis regarding the relative contributions of both routes. Only some results arising from experimental results (Al-Janabi et al., 1972; McPhie, 1974; Marciniszyn et al., 1976; Richter et al., 1999) have been reported. Fuentes et al. (2005b) made an individual- ised analysis for Scheme 3 whose validity and limitations are discussed below.

We have defined by means of equations (7), (8) and (10) three dimensionless kinetic parameters, $R_{u^{\prime}} R_{b}$ and $R$, any of them furnishing the instantaneous contribution of both the activation routes in Scheme 2 corresponding to the autocatalytic activation of a zymogen, including the formation of an active enzyme-zymogen complex. This reaction scheme (Fuentes et al., 2005a) is the most simple one which covers the main features described in the literature, i.e. a route of intramolecular activation of the zymogen into the active enzyme, $E$, and one or more peptides represented by $W$ [(route (a), Scheme 2] (Al-Janabi et al., 1972; Glick et al., 1982; Hayashi \& Sakamoto, 1986; Kageyama \& Takahashi, 1987; Tanaka \& Yada, 1997) and a route of autocatalytic activation of the zymogen by the active enzyme formed [route (b), Scheme 2] (Al-Janabi et al., 1972; Hayashi \& Sakamoto, 1986; Abad-Zapatero et al., 1990).

Route (a) of Scheme 2 condenses in a single step, the whole process corresponding to a conformational change of $Z$ molecules brought about by low $\mathrm{pH}$ and the subsequent cleavage of an N-terminal peptide. Thus, $k_{1}$ is actually an apparent rate constant corresponding to the whole process leading from $Z$ to $E$ and $W$ by intramolecular activation. Route (b) of Scheme 2 has been assumed to follow a single Michaelis-Menten mechanism instead of the more general Uni-Bi mechanism. This approach is the usual one used to describe mechanisms of autocatalytic zymogen activation and has been sufficiently justified (García-Moreno et al., 1991; Vázquez et al., 1993; Manjabacas et al., 1992; 1995).

The definition of $R_{u}$ for Scheme 2 given by equation (7) and its associated equations (3) and (5) are independent of the initial conditions and, therefore, of the presence or not at the onset of the reaction of the free enzyme E. In Fig. 2 we show the time course of $R_{u}$ under different initial conditions and in Table 1 we summarise the values of $\theta, t_{\infty}$ and $R_{u, \infty}$ corresponding to each of the curves. The following common features arise from Fig. 2 and Table 1:

(1) The time course of $R_{u}$ considerably differs according to the initial conditions, as expected.

(2) In all cases $R_{u}$ continuously decreases from $R_{u, 0}=1$ until a constant value, $R_{u, \infty}$, i.e. at any reaction time it is observed that $R_{u, 0} \geq R_{u} \geq R_{u, \infty}$.

(3) Since $\left[E_{T}\right]_{a}=0$ at $t=0$ and, therefore, when numerically determining $R_{u}$ an indetermination of type $0 / 0$ arises for $t=0$, it is easy to show that this indetermination is equal to unity [see equations (26) and (27)], i.e., it is always observed that $R_{u, 0}=1$ and, therefore, it is $[Z]_{0}$ - and $[E]_{0}$-independent.

(4) In contrast to $R_{u, 0^{\prime}} R_{u, \infty}$ is always $[Z]_{0}-$ and $[E]_{0}$-dependent.

(5) $R_{u, \infty}$ can be higher, equal or less than 0.5 
Table 1. Values of $\theta, t_{\infty}$ and $R_{u, \infty}$ corresponding to each of the time progress curves in Fig. 2.

For completeness on the 2 nd and 3rd columns we repeat the values of the initial zymogen and enzyme concentrations used for each of the curves in Fig. 2.

\begin{tabular}{llllll}
\hline Curve & {$[Z]_{0}(\mu \mathrm{M})$} & {$[E]_{0}(\mu \mathrm{M})$} & $\boldsymbol{\theta}(\mathbf{s})$ & $\boldsymbol{t}_{\infty}(\mathbf{s})$ & $\boldsymbol{R}_{u^{\prime} \infty}$ \\
\hline (A)-1 & 24 & 0 & 183 & 1859 & 0.2889 \\
$(\mathrm{~A})-2$ & 24 & 1 & 157 & 1855 & 0.2602 \\
$(\mathrm{~A})-3$ & 24 & 10 & 10.8 & 1840 & 0.0852 \\
$(\mathrm{~B})-1$ & 0.1 & 0.01 & - & 1906 & 0.5538 \\
$(\mathrm{~B})-2$ & 1 & 0.01 & 261 & 1865 & 0.3562 \\
$(\mathrm{~B})-3$ & 10 & 0.01 & 179 & 1432 & 0.2845 \\
\hline
\end{tabular}

(6) If $R_{u, \infty}>0.5$ (as it happens in curve 1 of Fig. 2B) then the predominant route is the intramolecular one, during the whole course of the reaction.

(7) If $R_{u, \infty}<0.5$ then there exists a time, which we will denote as $\theta$, at which the predominant route changes from the intra- to the intermolecular one.

(8) At a fixed $[Z]_{0}$ value $R_{u, \infty} t_{\infty}$ as well as $\theta$ decrease when $[E]_{0}$ increases.

(9) At a fixed $[E]_{0}$ value $R_{u, \infty} t_{\infty}$ as well as $\theta$ decrease when $[Z]_{0}$ increases.

\section{Extension of our analysis to other schemes of zymogen activation}

In this contribution we suggest a methodology of establishing the relative participation of both activation routes in zymogen activation evolving according to Scheme 2 from the previous knowledge of the values of the rate constants and initial conditions. This procedure, which is based either on the net active enzyme concentration or on the relative rate constants, is easily extrapolated to any other more or less complex enzyme reactions involving routes of intra- and intermolecular zymogen activation. In those cases in which no analytical expressions are possible for the whole time course of the species involved, as it occurs for example in Scheme 3, only a procedure, totally analogous to that used for Scheme 2, based on numerical integration of the corresponding set of differential equations describing the kinetic behaviour of the system is possible. On the contrary, if time course analytical expressions valid during the whole course of the reaction are possible for the species involved, as it happens for example in Scheme 1, then the numerical procedure can be replaced by another easier totally analytical one, based on the same general equations defining $R_{u}$. Next, as an example, we determine $R_{u}$ for the zymogen activation reaction evolving according to Schemes 3 and 1 .

Scheme 3

Proceeding for Scheme 3 analogously as we did for Scheme 2 we obtain the following expressions for $R_{u}$ :

$R_{u}=\frac{\left[E_{T}\right]_{u}}{[E]+[E Z]+[E S]-[E]_{0}}$ where $[E]_{u^{\prime}}[E],[E Z]$ and $[E S]$ are obtained by numerical integration of the set of differential equations:

$$
\begin{aligned}
\frac{d[E]}{d t} & =k_{1}[Z]-k_{2}[Z][E]-k_{4}[S][E]+ \\
& +\left(k_{-2}+2 k_{3}\right)[E Z]+\left(k_{-4}+k_{5}\right)[E S]
\end{aligned}
$$

$$
\frac{d[Z]}{d t}=-k_{1}[Z]-k_{2}[E][Z]+k_{-2}[E Z]
$$

$$
\frac{d[E Z]}{d t}=k_{2}[Z][E]-\left(k_{-2}+k_{3}\right)[E Z]
$$

$$
\frac{d[E S]}{d t}=k_{4}[S][E]-\left(k_{-4}+k_{5}\right)[E S]
$$

$$
\frac{d[S]}{d t}=-k_{4}[S][E]+k_{-4}[E S]
$$

$$
\frac{d\left[E_{T}\right]_{u}}{d t}=k_{1}[Z]
$$

Proceeding as for Scheme 2 one obtains that, for a very short reaction time, the same expressions for $R_{u}$ given by equation (26) and at $t=0$, equation (27) is also fulfilled.

Scheme 1

In this case $\left[E_{T}\right]_{a}$ is given by:

$\left[E_{T}\right]_{\mathrm{a}}=[E]-[E]_{0}$

and the system of differential equations describing the kinetic behaviour of the activation is:

$\frac{d[Z]}{d t}=-k_{1}[Z]-k_{2}[Z][E]$

$\frac{d[E]}{d t}=k_{1}[Z]+k_{2}[Z][E]$ 
For this simple reaction scheme, analytical expressions can be obtained for the time course of $Z$ and $E$. Effectively, from the mass balance we have: $[E]=[Z]_{0}+[E]_{0}-[Z]$

Hence, if equation (39) is inserted into equation (37) we have, after integration:

$$
[Z]=\frac{[Z]_{0} \lambda e^{-\lambda t}}{k_{1}+k_{2}[E]_{0}+k_{2}[Z]_{0} e^{-\lambda t}}
$$

where:

$$
\lambda=k_{1}+k_{2}\left([E]_{0}+[Z]_{0}\right)
$$

If in equation (40) we set $[E]_{0}=0$, the same expression for [Z] derived by Al-Janabi et al. (1972) is obtained, as expected, for Scheme 1 in the absence of enzyme at the onset of the reaction.

Hence, from equations (36) and (39) it results:

$$
\left[E_{T}\right]_{\mathrm{a}}=[Z]_{0}-[Z]
$$
we have:

If equation (40) is inserted into equation (42),

$$
\left[E_{T}\right]_{a}=\frac{\left(k_{1}+k_{2}[E]_{0}\right)[Z]_{0}\left(1-e^{-\lambda t}\right)}{k_{1}+k_{2}[E]_{0}+k_{2}[Z]_{0} e^{-\lambda t}}
$$

Hence, if in equation (35) we insert equation (40) and we solve the indicated integral, we obtain:

$$
\left[E_{T}\right]_{u}=\frac{k_{1}}{k_{2}} \ln \frac{\lambda}{k_{1}+k_{2}[E]_{0}+k_{2}[Z]_{0} e^{-\lambda t}}
$$

If now equations (43) and (44) are taken into account in equation (7), we have:

$$
\begin{gathered}
R_{u}=\frac{k_{1}\left(k_{1}+k_{2}[E]_{0}+k_{2}[Z]_{0} e^{-\lambda t}\right)}{k_{2}[Z]_{0}\left(k_{1}+k_{2}[E]_{0}\right)\left(1-e^{-\lambda t}\right)} . \\
\cdot \ln \frac{\lambda}{k_{1}+k_{2}[E]_{0}+k_{2}[Z]_{0} e^{-\lambda t}} .
\end{gathered}
$$

The above equation (45) is a rigorous analytical expression giving the $R_{u}$ value corresponding to the excessively simplified reaction mechanism in Scheme 1.

From equation (45) and (13), and the limit theory, it is easy to show, after solving the indeterminate type $\infty \cdot 0$, that $R_{u, 0}$ is given by:

$$
R_{u, 0}=\frac{k_{1}}{k_{1}+k_{2}[E]_{0}}
$$

Note that, on the contrary, for Schemes 2 and $3, R_{u, 0}$ is $[E]_{0}$-dependent and that if $[E]_{0}>0$ then $R_{u, 0}$ $<1$. Note also that $R_{u, 0}$ is higher, equal or less than
0.5 according to $k_{1}$ is higher, equal to or less than $k_{2}[E]_{0}$, respectively.

Likewise, from equation (45) and (14) we have:

$R_{u, \infty}=\frac{k_{1}}{k_{2}[Z]_{0}} \ln \frac{\lambda}{k_{1}+k_{2}[E]_{0}}$

$R_{u, \infty}$ given by equation (47) is, as expected, a quantity lying between 0 and 1 according to the values of the rate constants and initial concentrations.

From the meaning of $\eta$ [see equation (4)] and equation (39) the following relation between [Z] and $\eta$ exists:

$[Z]=(1-\eta)[Z]_{0}$

If now equation (48) is inserted into equation (40) it results in the following relationships giving the time $t$ needed for $\eta$ to reach a determined value:

$t=-\frac{1}{\lambda} \ln \frac{\left(k_{1}+k_{2}[E]_{0}\right)(1-\eta)}{k_{1}+k_{2}[E]_{0}+k_{2}[Z]_{0} \eta}$ duced to:

If $[E]_{0}=0$, then equations (45)-(49) become re-

$$
\begin{gathered}
R_{u}=\frac{k_{1}+k_{2}[Z]_{0} e^{-\left(k_{1}+k_{2}[Z]_{0}\right) t}}{k_{2}[Z]_{0}\left[1-e^{-\left(k_{1}+k_{2}[Z]_{0}\right) t}\right.} \cdot \\
\cdot \ln \frac{k_{1}+k_{2}[Z]_{0}}{k_{1}+k_{2}[Z]_{0} e^{-\left(k_{1}+k_{2}[Z]_{0}\right) t}} .
\end{gathered}
$$

$R_{u, 0}=1$

$$
\begin{aligned}
& R_{u, \infty}=\frac{k_{1}}{k_{2}[Z]_{0}} \ln \frac{k_{1}+k_{2}[Z]_{0}}{k_{1}} \\
& t=-\frac{1}{k_{1}+k_{2}[Z]_{0}} \ln \frac{k_{1}(1-\eta)}{k_{1}+k_{2}[Z]_{0} \eta}
\end{aligned}
$$

It is observed that the time course of $R_{u}$ could also be studied by numerical integration of the set of differential equations (37) and (38) in a similar way as it was done for Schemes 2 and 3. Nevertheless, when as in this case rigorous analytical solutions are possible, they are preferable due to their wider generality.

Another observation to be made is that Scheme 1 can formally be considered as a particular case of Scheme 2 when $k_{3} \rightarrow \infty$. Thus, all of the numerical and analytical treatment performed for Scheme 2 is also valid for Scheme 1. For example, if in equation (26) we set $k_{3} \rightarrow \infty$ it becomes equation (46). 
Table 2. Values of $k_{1}$ and $k_{2}$ obtained at different $\mathrm{pH}$ by Al-Janabi et al. (1972) for activation of pepsinogen to pepsin

\begin{tabular}{lll}
\hline $\mathrm{pH}$ & $\boldsymbol{k}_{1}\left(\mathrm{~min}^{-\mathbf{1}}\right)$ & $\boldsymbol{k}_{2}\left(\mathrm{~min}^{\mathbf{- 1}} \times \mathbf{m g} / \mathrm{ml}^{-\mathbf{1}}\right)$ \\
\hline 1.0 & $4.1 \pm 0.7$ & $1 \pm 0.2$ \\
2.0 & $2.6 \pm 0.2$ & $1.3 \pm 0.4$ \\
3.0 & $0.39 \pm 0.05$ & $2.6 \pm 0.6$ \\
4.0 & $0.008 \pm 0.0005$ & $0.20 \pm 0.03$ \\
\hline
\end{tabular}

Contribution of the intra- and intermolecular routes in pepsinogen autoactivation kinetics

Now, we apply some of the results obtained in the present paper to suggested models of pepsinogen activation involving one route of intramolecular activation and another one intermolecular (Koga \& Hayashi, 1976; Varón et al., 1994; Richter et al., 1998; Fuentes et al., 2005a; 2005b). In none of these contributions was any analysis allowing one to predict the contribution of the two activation routes
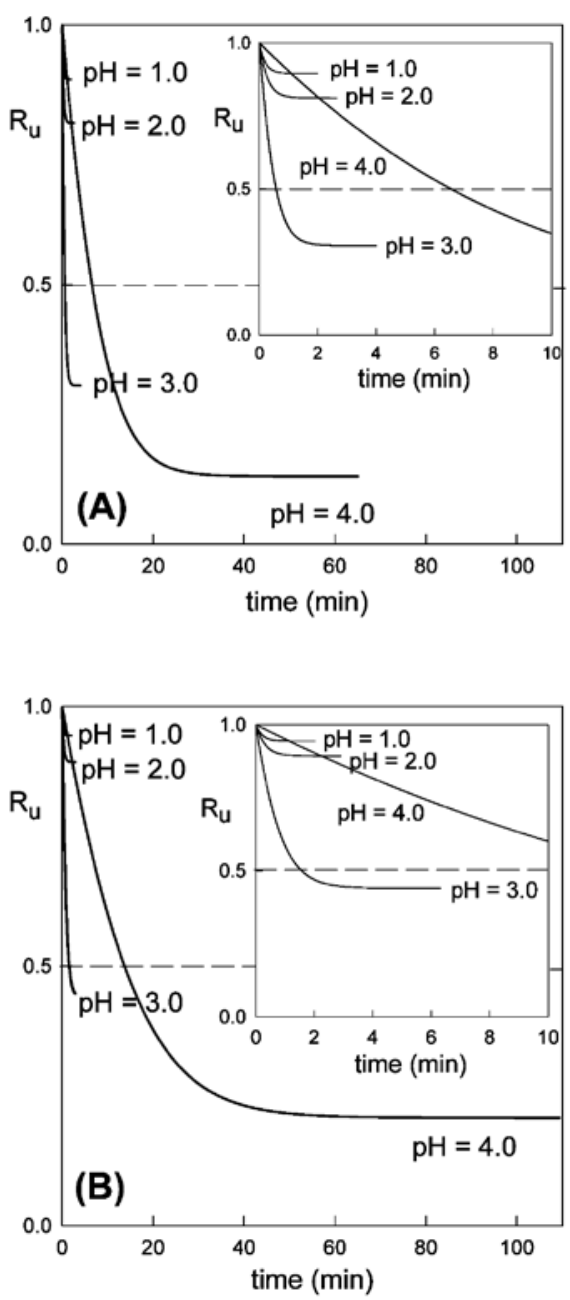

Figure 3. Time progress curves of $R_{u}$ for pepsinogen activation at the four different $\mathrm{pH}$ 's indicated and two different concentrations: (A) $1 \mathrm{mg} / \mathrm{ml}$ and (B) $0.5 \mathrm{mg} / \mathrm{ml}$.

The values of the rate constants $k_{1}$ and $k_{2}$ at each $\mathrm{pH}$ were as indicated in Table 2. The insets show details in the first $10 \mathrm{~min}$. from the values of the kinetic parameters and initial concentrations carried out. Only some conclusions based on the experimental results were made. Next we will apply our analysis to calculate the contribution of both activation routes involved in the pepsinogen activation carried out experimentally. For this task we will take the experimental results obtained by Al-Janaby (1972) and Fuentes et al. (2005a) concerning pepsinogen activation in the presence of only the zymogen at the onset of the reaction to better mimic the physiological conditions.

Al-Janabi (1972), by experimentally studying pepsinogen activation at different $\mathrm{pH}$ values and at $28^{\circ} \mathrm{C}$ and assuming a reaction mechanism as in Scheme 1, obtained the values of $k_{1}$ and $k_{2}$ shown in Table 2. However, he did not obtain any quantitative relationship of the contributions of both activating routes. In Fig. 3 we have plotted equation (50) corresponding to the time course of $R_{u}$ in the pepsinogen activation in the absence of pepsin at the onset of the reaction according to Scheme 1 using the rate constants values from Table 2 and two different initial zymogen concentrations.

In Table 3 we summarise the values of $\theta, t_{\infty}$ and $R_{u, \infty}$ corresponding to each of the curves in Fig. 3. Since $[E]_{0}=0$, according to equation (51) $R_{u, 0}$ $=1$ in all cases. From Fig. 3 and Table 3 the effect of the $\mathrm{pH}$ and initial pepsinogen concentration on the time course of $R_{u}$ and the values of $R_{u, \infty} \theta$ and $t_{\infty}$ are evident.

Recently, Fuentes et al. (2005a) proposed a reaction mechanism for pepsinogen activation in the absence of pepsin at the onset of the reaction according to Scheme 2 in which $Z$ is pepsinogen, $E$ is pepsin, $E Z$ the pepsin-zymogen complex and $W$ the peptide released from pepsinogen. These authors obtained, assuming a uniexponential kinetic behaviour, the values of the rate constants $k_{1}$ and $k_{3}$ and the Michaelis constant $K_{m}$ equal to $(5.14 \pm 0.56)$ $\times 10^{-3} \mathrm{~s}^{-1},(6.13 \pm 0.14) \times 10^{-4} \mathrm{~s}^{-1}$ and $(1.50 \pm 1.29)$ $\times 10^{-7} \mathrm{M}$, respectively, at $5^{\circ} \mathrm{C}$ and $\mathrm{pH}=2$. But the relative contribution of the intra- and intermolecular activation routes was not analysed in the mentioned contribution. Now, we can apply our results from this contribution for Scheme 2 under uniexponential behaviour to calculate the dependence of the time course of $R_{u}$ on the initial zymogen concentration for the same experimental conditions for which the rate constants and the Michaelis constant were evaluated. For the numerical integration of the set of differential equations (15)-(18) we need to know, apart from $[Z]_{0},[E]_{0}, k_{1}$ and $k_{3}$, the rate constants $k_{2}$ and $k_{-2}$. But the individual values of $k_{2}$ and $k_{-2}$ are not yet known, only their relationship through $K_{m}$ and $k_{3}$. Effectively, according to equation (1) it is observed that:

$k_{-2}=k_{2} K_{m}-k_{3}$ 
Table 3. Values of $\theta, t_{\infty}$ and $R_{u, \infty}$ corresponding to each of the time progress curves for $R_{u}$ in Fig. 3

\begin{tabular}{llllll}
\hline Curve & $\begin{array}{l}\text { Initial pepsinogen } \\
\text { concentration }(\mathbf{m g} / \mathbf{m l})\end{array}$ & $\mathbf{p H}$ & $\boldsymbol{R}_{u, \infty}$ & $\boldsymbol{\theta}(\mathbf{m i n})$ & $\boldsymbol{t}_{\infty}(\mathbf{m i n})$ \\
\hline (A)-1 & 1 & 1.0 & 0.8948 & - & 1.9900 \\
$(\mathrm{~A})-2$ & 1 & 2.0 & 0.8109 & - & 2.6440 \\
$(\mathrm{~A})-3$ & 1 & 3.0 & 0.3058 & 0.5782 & 3.9940 \\
$(\mathrm{~A})-4$ & 1 & 4.0 & 0.1303 & 6.5500 & 65.0000 \\
$(\mathrm{~B})-1$ & 0.5 & 1.0 & 0.9436 & - & 2.0300 \\
$(\mathrm{~B})-2$ & 0.5 & 2.0 & 0.8926 & - & 2.9100 \\
(B)-3 & 0.5 & 3.0 & 0.4339 & 1.5730 & 6.3200 \\
(B)-4 & 0.5 & 4.0 & 0.2082 & 13.7125 & 109.4000 \\
\hline
\end{tabular}

Equation (54) shows that there is a minimal $k_{2}$ value (corresponding to $k_{-2}=0$ ) equal to $k_{3} / K_{m}$ equal to $4.0867 \times 10^{3} \mathrm{~s}^{-1}$ in this case, and that the higher $k_{2}$ values determine the corresponding $k_{-2}$ values.

In Fig. 4 we show the time course of $R_{u}$ using for $k_{1}$ and $k_{3}$ their above-indicated values and for $[Z]_{0}, k_{2}$ and $k_{-2}$ the values shown in Table 4 . The value $k_{2}=1 \times 10^{9}$ has been included in Table 4 to have the values of $t_{\infty}$, and, in its case, of $\theta$, when $k_{2} \rightarrow \infty$, but no corresponding plot is drawn in Fig. 4. From Fig. 4 and Table 4 the following results are evident: (1) For a fixed $[Z]_{0}$ value $t_{\infty}$ increases when $k_{2}$ decreases; (2) For a fixed $k_{2}$ value $R_{u, \infty}$ increases when $[Z]_{0}$ increases; (3) For a fixed $k_{2}$ value $R_{u}$ at any time increases and $t_{\infty}$ decreases when $[Z]_{0}$ decreases; (4) Great differences in the $k_{2}$ values produce only very small differences in the $R_{u}$ values at any reaction time; (5) Only for high $[Z]_{0}$ values there is a transition between the predominant routes from

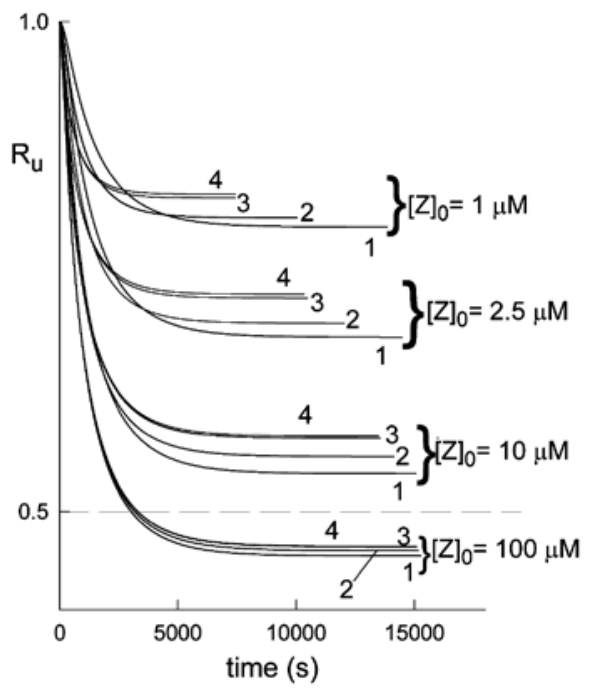

Figure 4. Different time progress curves for $R_{u}$ corresponding to $K_{m}=1.5 \times 10^{-7} \mathrm{M}$ and a fixed initial pepsinogen concentration $[Z]_{0}$ but different $k_{2}$-values.

The values of $k_{2}$ were $4.0867 \times 10^{3} \mathrm{M}^{-1} \mathrm{~s}^{-1}$ in curves $1,1.000 \times 10^{4} \mathrm{M}^{-1} \mathrm{~s}^{-1}$ in curves $2,1.000 \times 10^{5} \mathrm{M}^{-1} \mathrm{~s}^{-1}$ in curves 3 and $1.000 \times 10^{6} \mathrm{M}^{-1} \mathrm{~s}^{-1}$ in curves 4 . The fixed initial pepsinogen concentration used in the simulations was as indicated. The values of the rate constants used for each time progress curve $R_{u}$ were: $k_{1}=5.14 \times 10^{-3} \mathrm{~s}^{-1}$ and $k_{3}=6.13 \times 10^{-4} \mathrm{~s}^{-1}$. the intra- to the intermolecular one; (6) In the case of transition of both routes the above-defined time $\theta$ increases when $k_{2}$ increases at a fixed $[Z]_{0}$ value; (7) At any fixed $[Z]_{0}$ value a minimal $R_{u, \infty}$ is reached when $k_{2} \rightarrow \infty$; (8) At any fixed $[Z]_{0}$ value, the difference between the $R_{u, \infty}$ values corresponding to the minimal $k_{2}$ value and $k_{2} \rightarrow \infty$ is small; (9) For any $[Z]_{0}$ value, Fig. 4 and Table 4 allow one to establish the upper and lower limits for the $R_{u}$ value at any time that correspond to the minimal $k_{2}$ value and $k_{2}$ $\rightarrow \infty$.

Suggestion of a procedure for the evaluation of the individual values of $k_{2}$ and $k_{-2}$

Obviously, if the $k_{2}$ and $k_{-2}$ values could be known, then only one plot of $R_{u}$ would exist for each $[Z]_{0}$ concentration. But our analysis allows us to suggest the experimental workers a procedure to estimate these two constants from experimental data under any experimental conditions for any zymogen activation fitting the model in Scheme 2. This procedure could consist of the following steps: (a) Evaluation of the values for the rate constants $k_{1}$ and $k_{3}$ and $K_{m}$ as made by Fuentes et al. (2005a); (b) For

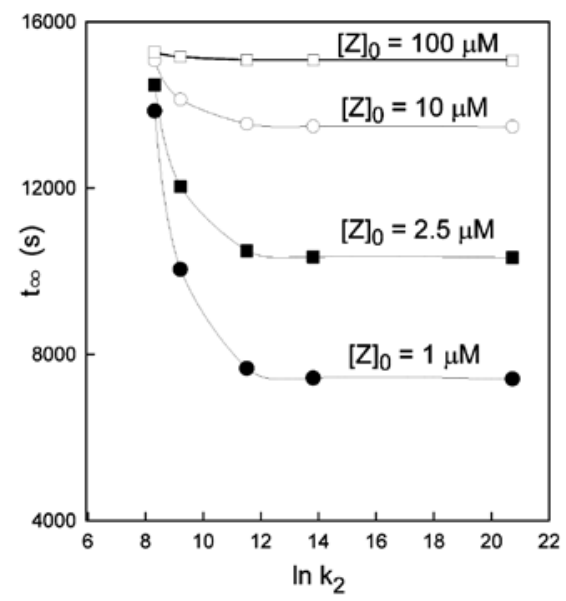

Figure 5. Plots of $t_{\infty} v s \ln k_{2}$ from Table 5 for each of the corresponding pepsinogen concentrations.

The pepsinogen concentrations used were: $1 \mu \mathrm{M}(\bullet), 2.5$ $\mu \mathrm{M}(\mathbf{\square}), 10 \mu \mathrm{M}(\mathrm{O})$, and $100 \mu \mathrm{M}(\square)$. The solid lines are plots of equation (55) to which the data were fitted. The values of $a, b$ and $c$ are summarised in Table 5 . 
Table 4. Values of $k_{2}, k_{-2}, \theta, R_{u, \infty}$ and $t_{\infty}$ corresponding to each curve in Fig. 4.

The value of $k_{2}=1 \times 10^{9}$ and the corresponding one of $k_{-2}$ were added to have the limit values of $R_{u, \infty}$ and $t_{\infty}$ at high $k_{2}$ values. Note that $K_{m}=1.5 \times 10^{-7} \mathrm{M}$ in all cases.

\begin{tabular}{|c|c|c|c|c|c|c|}
\hline$[Z]_{0} \quad(\mu \mathrm{M})$ & Curve & $k_{2}\left(\mathrm{M}^{-1} \mathrm{~s}^{-1}\right)$ & $k_{-2}\left(\mathrm{~s}^{-1}\right)$ & $\theta(\mathrm{s})$ & $R_{u, \infty}$ & $t_{\infty}$ \\
\hline 1 & 1 & $4.0867 \times 10^{3}$ & $5.0033 \times 10^{-9}$ & - & 0.7906 & 13853 \\
\hline 1 & 2 & $1.0000 \times 10^{4}$ & $8.8700 \times 10^{-4}$ & - & 0.7999 & 10041 \\
\hline 1 & 3 & $1.0000 \times 10^{5}$ & $1.4387 \times 10^{-2}$ & - & 0.8200 & 7658 \\
\hline 1 & 4 & $1.0000 \times 10^{6}$ & 0.149387 & - & 0.8239 & 7426 \\
\hline 1 & - & $1.0000 \times 10^{9}$ & 150.00 & - & 0.8244 & 7404 \\
\hline 2.5 & 1 & $4.0867 \times 10^{3}$ & $5.0033 \times 10^{-9}$ & - & 0.6782 & 14481 \\
\hline 2.5 & 2 & $1.0000 \times 10^{4}$ & $8.8700 \times 10^{-4}$ & - & 0.6924 & 12035 \\
\hline 2.5 & 3 & $1.0000 \times 10^{5}$ & $1.4387 \times 10^{-2}$ & - & 0.7180 & 10488 \\
\hline 2.5 & 4 & $1.0000 \times 10^{6}$ & 0.149387 & - & 0.7218 & 10342 \\
\hline 2.5 & - & $1.0000 \times 10^{9}$ & 150.00 & - & 0.7223 & 10326 \\
\hline 10 & 1 & $4.0867 \times 10^{3}$ & $5.0033 \times 10^{-9}$ & - & 0.5388 & 15065 \\
\hline 10 & 2 & $1.0000 \times 10^{4}$ & $8.8700 \times 10^{-4}$ & - & 0.5563 & 14129 \\
\hline 10 & 3 & $1.0000 \times 10^{5}$ & $1.4387 \times 10^{-2}$ & - & 0.5751 & 13542 \\
\hline 10 & 4 & $1.0000 \times 10^{6}$ & 0.149387 & - & 0.5773 & 13485 \\
\hline 10 & - & $1.0000 \times 10^{9}$ & 150.00 & - & 0.5778 & 13475 \\
\hline 100 & 1 & $4.0867 \times 10^{3}$ & $5.0033 \times 10^{-9}$ & 3031 & 0.4548 & 15274 \\
\hline 100 & 2 & $1.0000 \times 10^{4}$ & $8.8700 \times 10^{-4}$ & 3178 & 0.4602 & 15154 \\
\hline 100 & 3 & $1.0000 \times 10^{5}$ & $1.4387 \times 10^{-2}$ & 3310 & 0.4641 & 15085 \\
\hline 100 & 4 & $1.0000 \times 10^{6}$ & 0.149387 & 3325 & 0.4647 & 15077 \\
\hline 100 & - & $1.0000 \times 10^{9}$ & 150.00 & 3354 & 0.4653 & 15074 \\
\hline
\end{tabular}

any experimental value of initial zymogen concentration, experimental determination of the time the reaction takes to complete the activation of $\mathrm{Z}$ into $\mathrm{E}$, i.e. $t_{\infty, \text { exp }}$ (c) Using this initial value of the zymogen concentration and those of $k_{1}, k_{3}$ and $K_{m^{\prime}}$ plotting of curves corresponding to different values of $k_{2}$, like curves 1, 2, 3 and 4 in Fig. 4; (d) From the above plots, drawing, in a figure like Fig. 5, the different $t_{\infty}$-values vs $k_{2}$; (e) Then, using this plot, determinination of the $k_{2}$ value corresponding to $t_{\infty, \text { exp }}$.

For step (e) either one interpolates or one fits the different points to an equation, e.g. a uniexponential one. In our Fig. 4 the points corresponding to each zymogen concentration fit very well the single exponential decay three parameters equation:

$t_{\infty}=a+b e^{-c \ln k_{2}}$

where the values of $a, b$ and $c$ are given in Table 5 . Thus, Fuentes et al. (2005a) could have evaluated $k_{2}$ and $k_{-2}$ by merely measuring $t_{\infty, \exp }$ for any initial pepsinogen concentration if they had the present analysis.

Previous attempt of an analytical expression to evaluate the contribution of the intra- and intermolecular activation of pepsinogen

Recently, Fuentes et al. (2005b) studied, both theoretically and experimentally, pepsinogen activation according to Scheme 3 at $\mathrm{pH}=3.0$ and $25^{\circ} \mathrm{C}$ and assuming rapid equilibrium in the two reversible steps. They derived time course equations which were valid as long as the instantaneous pepsinogen concentration did not considerably differ (by no more than $30 \%$ ) from its initial concentration. As part of their work, they suggest a partition ratio, $r$, to determine the relative contribution of the intra- and intermolecular routes. They defined $r$ as the quotient between the rate of instantaneous formation of $[E]$ in route (a) in Scheme 3 and the rate of formation of $[E]$ from $[E Z]$ in route $(b)$, i.e.:

$r=\frac{k_{1}[Z]}{2 k_{3}[E Z]}$

and then, in equation (56) they substituted [Z] by $[Z]_{0}$ and $[E Z]$ by their corresponding equations, obtaining:

$$
r=\frac{k_{1}[Z]_{0}}{2 k_{3}\left(e^{\frac{k_{3} K_{4}[Z]_{0}}{K_{4}[Z]_{0}+K_{2}[S]_{0}+K_{2} K_{4}}}-1\right)}
$$

where $[S]_{0}$ is the initial concentration of the substrate $S, K_{2}$ is given by equation (2) and $K_{4}$ is the equilibrium dissociation constant of the complex $E S$ equal to $k_{-4} / k_{4}$. Depending on whether $r>1, r=1$ 
Table 5. Values of $a, b$ and $c$ obtained from fitting of the data in Fig. 4 to equation (55).

In the 4th column the norm of each of the fittings is given.

\begin{tabular}{lllll}
\hline$[Z]_{0}(\mu \mathrm{M})$ & $\begin{array}{l}\boldsymbol{a} \\
(\mathrm{s})\end{array}$ & $\boldsymbol{b}$ & $\boldsymbol{c}$ & $\begin{array}{l}\text { norm } \\
(\mathrm{s})\end{array}$ \\
\hline 1 & $7.400 \times 10^{3} \pm 4.171 \times 10^{-13}$ & $2.261 \times 10^{7} \pm 6.968 \times 10^{-8}$ & $9.993 \times 10^{-1} \pm 3.193 \times 10^{-6}$ & $9.095 \times 10^{-13}$ \\
2.5 & $1.032 \times 10^{4} \pm 4.328$ & $1.608 \times 10^{7} \pm 6.832 \times 10^{5}$ & $9.933 \times 10^{-1} \pm 5.103 \times 10^{-3}$ & 9.419 \\
10 & $1.348 \times 10^{4} \pm 1.112$ & $6.193 \times 10^{6} \pm 1.773 \times 10^{5}$ & $9.943 \times 10^{-1} \pm 3.438 \times 10^{-3}$ & 2.421 \\
100 & $1.508 \times 10^{4} \pm 1.227$ & $1.035 \times 10^{6} \pm 2.733 \times 10^{5}$ & $1.030 \pm 3.173 \times 10^{-2}$ & 2.699 \\
\hline
\end{tabular}

or $r<1$, the unimolecular route will prevail over the bimolecular route, both routes will be balanced, or the bimolecular route prevail over the unimolecular route, respectively.

Equation (57) was the first analytical equation proposed to establish the relative contributions of both activation steps in pepsinogen activation. On the basis of the present contribution we can offer the following comments regarding its limitations:

(1) Equation (57) is only applicable at the onset of the reaction, when the kinetic equations derived by Fuentes et al. (2005b) are valid, and not later into the reaction.

(2) $2 k_{3}[E Z]$ is not the rate of $E$ formation in the bimolecular route. Effectively, equation (30) gives the total rate of formation of $E$ including both the uni- and bimolecular routes. If now we subtract from this rate that in the unimolecular route, $k_{1}[Z]$, it results in:

$$
\begin{array}{r}
\left(\frac{d[E]}{d t}\right)_{\text {bimolecular route }}=-k_{2}[Z][E]-k_{4}[S][E]+ \\
+\left(k_{-2}+2 k_{3}\right)[E Z]+\left(k_{-4}+k_{5}\right)[E S]
\end{array}
$$

which considerably differs from $2 k_{3}[E Z]$.

(3) The definition of $r$ is only given for Scheme 3 and no general validity was assumed.

\section{CONCLUDING REMARKS}

Proteolytic enzymes play an important role in the life cycle of proteins. From a kinetic point of view, autocatalytic zymogen activation processes are harder to study since in these cases the activating and the activated enzyme are the same entity. In the present study we have derived for the first time a dimensionless distribution coefficient allowing one to predict whether the uni- or the bimolecular reaction prevails in the activation process under the experimental conditions used once values are given to the kinetic parameters involved in the system. This coefficient, $R_{u}$, relates, with the reaction time and the initial zymogen concentration, the relative weight of the inter- and intramolecular routes in any zymogen activation process when its kinetic behaviour can be described by Scheme 2, which includes the zymogen intramolecular activation step, the intermolecular activation step between the zymogen and the active enzyme and takes into account the formation of an active enzyme-zymogen complex. The results obtained can be extrapolated to many other zymogen autoactivation reactions involving intra- and intermolecular activation routes such as those in Schemes 1 and 3, thus being in our opinion a very useful tool in the kinetic study of limited proteolysis reactions, key in metabolic regulation. In this way, we have obtained for the first time the time course of $Z$ and $E$ in a zymogen activation process evolving according to Scheme 1 when the activated enzyme (which coincides with the activating one) is present at the onset of the reaction.

The results obtained have been applied to the pepsinogen activation process according to Schemes 1 and 2 using the values of the rate and equilibrium constants from the literature, giving for the first time the time course of the contribution of both routes for any initial values of the pepsinogen and pepsin concentrations. Our results include some data predicted, without any theoretical support, by other authors (Al-Janabi et al., 1972; McPhie, 1974; Marciniszyn et al., 1976; Richter et al., 1999), thus both Figs. 3 and 5 involve data reported by Al-Janabi et al. (1972), who indicated that below $\mathrm{pH} 3.0$ and at concentrations under $1 \mathrm{mg} / \mathrm{ml}(24.4 \mu \mathrm{M})$, pepsinogen activates predominantly by means of an intramolecular mechanism, and they also agree with Kageyama and Takahashi (1987), who indicated that the intramolecular reaction is especially important in the initial phase of the activation process to generate the active enzyme and that the intermolecular reaction plays a significant role in acceleration and completion of the activation.

It is evident that all active enzyme molecules obtained by the intra- and intermolecular routes are equal. However, the kinetics of the two pathways and so their dynamic behaviour is very different, since the first of them is a unimolecular reaction, whereas the second is a bimolecular process. The predominance of one route or the other is dependent upon the experimental conditions: $\mathrm{pH}$, zymogen concentration, initial presence of pepsin, etc. (Al-Janabi et al., 1972; Mc-Phie, 1974; Kageyama \& Takahashi, 1987). According to van Hazel et al. (1997), to maintain strict subcellular compartmentalization of 
hydrolytic activities, efficient regulation of autoactivation is required. Thus there are two counteracting demands: the threshold for intracellular autoactivation must be low enough to allow this process to take place, but high enough to avoid premature enzymatic activity. Therefore, the knowledge of the predominant activation pathway represents a useful tool in the study of the physiological conditions of the activation process of a zymogen at intra- and extracellular levels, as well as in the study of possible regulation mechanisms of autocatalytic zymogen activation processes.

Not all zymogen activations proceed through the simultaneous routes of uni- and bimolecular activations. Many zymogen activation processes are only bimolecular (Galindo et al., 1982; Varón et al., 1991; 1992; Vázquez et al., 1993; Manjabacas et al., 1996; Wu et al., 2001). The coexistence of intra- and intermolecular activation pathways is a feature of aspartic proteinases, e.g. pepsinogen and proproteinase A. Moreover, with regard to the activation of aspartic proteinase zymogens, several mechanisms are possible in physiological conditions, including those shown in this paper as examples (Foltman, 1966; Pedersen et al., 1979; Kageyama, 1988; Chou \& Howe, 2002), but all of them showing the two above-mentioned activation pathways. All the mechanisms of activation of aspartic proteinases can be treated using the analysis suggested here.

Based on our analysis of Scheme 2 a procedure is suggested that, from the results of Fuentes et al. (2005a) and the measurement of the time the activation process takes to completely activate pepsinogen, allows the evaluation of $k_{2}$ and $k_{-2}$ from $k_{1}$, $k_{3}$ and $K_{2}$.

Finally, a previous attempt to analytically quantify the relative contribution of both the uniand bimolecular routes in pepsinogen activation (Fuentes et al., 2005b) has been critically reviewed.

\section{Acknowledgements}

This work was supported by grants from the Comisión Interministerial de Ciencia y Tecnología (MCyT, Spain), Project No. BQU2002-01960 and from Junta de Comunidades de Castilla-La Mancha, Project No. PAI-05-036.

\section{REFERENCES}

Abad-Zapatero C, Rydel TJ, Erickson J (1990) Revised 2.3 A structure of porcine pepsin: evidence for a flexible subdomain. Proteins 8: 62-81.

Al-Janabi J, Hartsuck JA, Tang J (1972) Kinetics and mechanism of pepsinogen activation. J Biol Chem 247: 46284632.

Boatright KM, Salvesen GS (2003) Mechanism of caspase activation. Curr Opin Cell Biol 15: 725-731.
Chen JM, Kukor Z, Le Marechal C, Coth M, Tsakiris L, Raguenes C, Ferec C, Sahin-Toth M (2003) Evolution of trypsinogen activation peptides. Mol Biol Evol 20: 1767-1777.

Chou K-C, Howe WJ (2002) Prediction of the tertiary structure of the $\beta$-secretase zymogen. Biochem Biophys Res Commun 292: 702-708.

Fehlberg EK (1970) Formeln vierter und niedrigerer Ordnung mit Schrittweiten-Kontrolle und ihre Anwendung auf Wärmeleitungsprobleme. Computing 6: 61-71.

Foltmann B (1966) A review on prorennin and rennin. CR Trav Lab Carlsberg 35: 143-151.

Fuentes ME, Varón R, García-Moreno M, Valero E (2005a) Kinetics of intra- and intermolecular zymogen activation with formation of an enzyme-zymogen complex. FEBS J 272: 85-96.

Fuentes ME, Varón R, García-Moreno M, Valero E (2005b) Kinetics of autocatalytic zymogen activation measured by a coupled reaction: pepsinogen autoactivation. Biol Chem 386: 689-698.

Galindo JD, Peñafiel R, Varón R, Pedreño E, García-Carmona F, García-Cánovas F (1983) Kinetic study of the activation process of frog epidermis pro-tyrosinase by trypsin. Int J Biochem 15: 633-637.

García-Moreno M, Havsteen BH, Varón R, Rix-Matzen H (1991) Evaluation of the kinetic parameters of the activation of trypsinogen by trypsin. Biochim Biophys Acta 1080: 143-147.

García-Sevilla F, Garrido del Solo C, Duggleby RG, GarcíaCánovas F, Peyró R, Varón R (2000) Use of a windows program for simulation of the progress curves of reactants and intermediates involved in enzyme-catalyzed reactions. Biosystems 54: 151-164.

Glick DM, Valler MJ, Rowlands CC, Evans JC, Kay J (1982) Activation of spin-labeled chicken pepsinogen. Biochemistry 16: 3746-3750.

Glick DM, Shalitin Y, Hilt CR (1989) Studies on the irreversible step of pepsinogen activation. Biochemistry 28: 2626-2630.

Hayashi K, Sakamoto N (1986) Determination of reaction scheme and kinetic parameters. In Dynamic Analysis of Enzyme Systems. An Introduction. pp 297-303, Japan Scientific Societies Press, Tokyo.

Kageyama T (1988) Analysis of the activation of pepsinogen in the presence of protein substrates and estimation of the intrinsic proteolytic activity of pepsinogen. Eur J Biochem 176: 543-549.

Kageyama T, Takahashi K (1987) Activation mechanism of monkey and porcine pepsinogens A. One-step and stepwise activation pathways and their relation to intramolecular and intermolecular reactions. Eur J Biochem 165: 483-490.

Kanost MR, Jiang H, Yu XQ (2004) Innate immune responses of a lepidopteran insect, Manduca sexta. Immunol Rev 198: 97-105.

Koga D, Hayashi K (1976) Activation process of pepsinogen. J Biochem 5: 449-476.

Lin X, Lin Y, Koelsch G, Gustchina A, Wlodawer A, Tang J (1992) Enzymic activities of two-chain pepsinogen, two-chain pepsin, and the amino-terminal lobe of pepsinogen. J Biol Chem 267: 17257-17263.

Liu JH, Wang ZX (2004) Kinetic analysis of ligand-induced autocatalytic reactions. Biochem J 379: 697-702.

Lluis F, Roma J, Suelves M, Parra M, Aniorte G, Gallardo E, Illa I, Rodríguez L, Hughes SM, Carmeliet P, Roig M, Muñoz-Cánoves P (2001) Urokinase-dependent plasminogen activation is required for efficient skeletal muscle regeneration in vivo. Blood 97: 1703-1711. 
Magklara A, Mellati AA, Wasney GA, Little SP, Sotiropoulou G, Becker GW, Diamandis EP (2003) Characterization of the enzymatic activity of human kallikrein 6: autoactivation, substrate specificity, and regulation by inhibitors. Biochem Biophys Res Commun 307: 948-955.

Manjabacas MC, Valero E, García-Moreno M, García-Cánovas F, Rodríguez JN, Varón R (1992) Kinetic analysis of the control through inhibition of autocatalytic zymogen activation. Biochem J 282: 583-587.

Manjabacas MC, Valero E, García-Moreno M, Varón R (1995) Kinetic analysis of an autocatalytic process coupled to a reversible inhibition. The inhibition of the system trypsinogen-trypsin by $p$-aminobenzamidine. Biol Chem Hoppe-Seyler 376: 577-580.

Manjabacas MC, Valero E, García-Moreno M, Garrido C, Varón R (1996) Kinetics of an autocatalytic zymogen reaction in the presence of an inhibitor coupled to a monitoring reaction. Bull Math Biol 58: 19-41.

Manjabacas MC, Valero E, Moreno-Conesa M, GarcíaMoreno M, Molina-Alarcón M, Varón R (2002) Linear mixed irreversible inhibition of the autocatalytic activation of zymogens. Kinetic analysis checked by simulated progress curves. Int J Biochem Cell Biol 34: 358-369.

Marciniszyn J Jr, Huang JS, Hartsuck JA, Tang J (1976) Mechanism of intramolecular activation of pepsinogen. Evidence for an intermediate delta and the involvement of the active site of pepsin in the intramolecular activation of pepsinogen. J Biol Chem 254: 7095-7102.

Marin F, Roldan V, Lip GY (2003) Fibrinolytic function and atrial fibrillation. Thromb Res 109: 233-240.

Mathews JH, Fink KD (1999) Ecuaciones diferenciales ordinarias. In Métodos Numéricos con MATLAB 3rd edn, (Capella I, ed) pp 505-509, Prentice Hall, Madrid, Spain.

McKay TR, Bell S, Tenev T, Stoll V, Lopes R, Lemoine NR, McNeish IA (2003) Procaspase 3 expression in ovarian carcinoma cells increases surviving transcription which can be countered with a dominant-negative mutant, surviving T34A; a combination gene therapy strategy. Oncogene 22: 3539-3547.

McPhie P (1974) Pepsinogen: activation by a unimolecular mechanism. Biochem Biophys Res Commun 56: 789-792.

Pearton DJ, Nirunsuksiri W, Rehemtulla A, Lewis SP, Presland RB, Dale BA (2001) Proprotein convertase expression and localization in epidermis: evidence for multiple roles and substrates. Exp Dermatol 10: 193-203.

Pedersen VB, Christensen KA, Foltmann B (1979) Investigations on the activation of bovine prochymosin. Eur J Biochem 94: 573-580.
Richter C, Tanaka T, Yada RY (1998) Mechanism of activation of the gastric aspartic proteinases: pepsinogen, progastricsin and prochymosin. Biochem J 335: 481-490.

Richter C, Tanaka T, Koseki T, Yada RY (1999) Contribution of a prosegment lysine residue to the function and structure of porcine pepsinogen $\mathrm{A}$ and its active form pepsin A. Eur J Biochem 261: 746-752.

Shariat-Madar Z, Mahdi F, Schmaier AH (2004) Recombinant prolylcarboxypeptidase activates plasma prekallikrein. Blood 103: 4554-4561.

Shi Y (2004) Caspase activation: revising the induced proximity model. Cell 117: 855-858.

Spronk HM, Govers-Fiemslag JW, ten Cate H (2003) The blood coagulation system as a molecular machine. Bioessays 25: 1220-1228.

Tanaka T, Yada RY (1997) Engineered porcine pepsinogen exhibits dominant unimolecular activation. Arch Biochem Biophys 340: 355-358.

van den Hazel HB, Wolff AM, Kielland-Brandt MC, Winther JR (1997) Mechanism and ion-dependence of in vitro autoactivation of yeast proteinase A: possible implications for compartmentalized activation in vivo. Biochem J 326: 339-344.

Varón R, Román A, García-Canovas F, García-Carmona F (1986) Transient phase kinetics of activation of humanplasminogen. Bull Math Biol 48: 149-166.

Varón R, Havsteen BH, Vázquez A, García-Moreno M, Valero E, García-Cánovas F (1990) Kinetics of the trypsinogen activation by enterokinase and trypsin. $J$ Theor Biol 145: 123-131.

Varón R, Havsteen BH, García-Moreno M, Vázquez A, Tudela J, García-Cánovas FG (1991) Kinetics of the trypsinogen activation by enterokinase and or trypsincoupling of a reaction in which the trypsin acts on one of its substrates. J Mol Catal 66: 409-419.

Varón R, Havsteen BH, García-Moreno M, Vázquez A (1992) Kinetics of a model of autocatalysis, coupling of a reaction in which the enzyme acts on one of its substrates. J Theor Biol 154: 261-270.

Varón R, Molina R, García-Moreno M, García-Sevilla F, Valero E (1994) Kinetic analysis of the opened bicyclic enzyme cascades. Biol Chem Hoppe-Seyler 375: 365-371.

Vázquez A, Varón R, Tudela J, García-Cánovas F (1993) Kinetic characterization of a model for zymogen activation: an experimental design and kinetic data analysis. J Mol Catal. 79: 347-363.

Wu J-W, Wu Y, Wang Z-X (2001) Kinetic analysis of a simplified scheme of autocatalytic zymogen activation. Eur J Biochem 268: 1547-1553. 